Aim of the study: To evaluate prevalence of rebound thymic hyperplasia (RTH) after bone marrow transplantation (BMT) in paediatric patients with haemato-oncological diseases.

Material and methods: Between February 2013 and December 2017, BMT was performed in 189 paediatric patients with haemato-oncological diseases in our institution. Fifty-six patients who underwent at least two chest computed tomography (CT) exams performed before and after BMT were included in the study. Maximum transverse and anterior-posterior (AP) diameters and $C T$ attenuation of the thymus were measured on axial images. Thymic enlargement was considered when both transverse and AP diameters increased. RTH was defined as the presence of thymic enlargement on $\mathrm{CT}$ after BMT relative to the CT taken before.

Results: Twenty of 56 patients (36\%) demonstrated RTH (12 boys, 8 girls; age range $=4-18$ years; median age $=9.8$ years). In 20 patients with RTH, seven patients (35\%) were diagnosed with ALL, five patients (25\%) with thalassemia, two patients (10\%) with $\mathrm{AML}$, and one patient (5\%) with various diseases. Mean follow-up period between pre-BMT CT and BMT was 46 days, which was 239 days between BMT and post-BMT CT. Mean thymic transverse and $\mathrm{AP}$ diameters were $9 \mathrm{~mm}$ and $16 \mathrm{~mm}$, respectively, before BMT, which were $17 \mathrm{~mm}$ and $33 \mathrm{~mm}$ after BMT. Mean HU was 57 on contrast enhanced and 35 on unenhanced images before BMT, which were 59 and 36, respectively, after BMT.

Conclusions: RTH is common finding after BMT in children with various haemato-oncological diseases and should be taken under consideration in paediatric patients after BMT.

Key words: thymus, rebound hyperplasia, haemato-oncological disease, computed tomography.

Contemp Oncol (Pozn) 2018; 22 (2): 95-98 DOI: https://doi.org/10.5114/wo.2018.75398

\section{Rebound thymic hyperplasia after bone marrow transplantation in children with haemato-oncological diseases}

\author{
Taner Arpaci $^{1}$, Barbaros Sahin Karagun ${ }^{2}$
}

${ }^{1}$ Department of Radiology, Acibadem Adana Hospital, Acibadem University, Adana, Turkey

${ }^{2}$ Department of Paediatric Haematology and Oncology, Acibadem Adana Hospital, Acibadem University, Adana, Turkey

\section{Introduction}

The thymus gland is a lymphoid organ that is located in the anterior mediastinum and essential for foetal development and maturation of T lymphocytes [1]. It has bilobulated configuration and may represent as pyramidal, triangular, or quadrilateral shape on imaging [2, 3]. The thymus approaches its highest dimensions at puberty and then undergoes involution [4]. Thymic hyperplasia includes two subgroups: true hyperplasia and lymphoid hyperplasia. True thymic hyperplasia is described as enlargement of the gland in terms of weight and volume. It emerges in patients who are recovering from a stress such as chemotherapy, radiotherapy, bone marrow transplantation, corticosteroid therapy, thermal burns, operations, and pneumonia. As a response to the stress, the thymus first becomes smaller and then enlarges when the stress disappears. It may become larger than its original dimensions; this entity is defined as "rebound hyperplasia" [4, 5]. In patients with rebound thymic hyperplasia $(\mathrm{RTH})$, the thymus re-establishes peripheral T lymphocytes and immune function after chemotherapy-induced lymphopaenia [6]. Some authors suggest that RTH developing after chemotherapy indicates a good prognosis [1]. RTH mostly happens within the first year following chemotherapy. It is usually observed in children but may also occur in young adults [1]. Lymphoid hyperplasia of the thymus is defined as an increased number of lymphoid follicles in the gland and it may be with atrophy or enlargement of the thymus. Lymphoid hyperplasia is associated with autoimmune diseases like myasthenia gravis, thyrotoxicosis, or neoplastic lymphoid infiltration $[4,5]$. The purpose of this study was to evaluate the prevalence of RTH after bone marrow transplantation (BMT) in paediatric patients with haemato-oncological diseases.

\section{Material and methods}

Between February 2013 and December 2017, bone marrow transplantation (BMT) was performed in 189 paediatric patients with different haemato-oncological diseases in our institution. Fifty-six patients who underwent at least two chest computed tomography (CT) exams for any reason performed before and after BMT, respectively, were included in the study. A dual-source, 64-slice CT scanner (Somatom Definition/AS $64 \times 2$ : Siemens Medical Systems, Erlangen, Germany) was used in the study. The CT images were retrospectively reviewed by a blinded radiologist experienced in thoracic imaging. Maximum transverse and anterior-posterior (AP) diameters of the thymus were measured on axial images. Also, CT attenuation of the thymus was measured using an oval region of interest (ROI), which covered only the thymus gland excluding surrounding fat on axial images. The measurements were performed by using 
a PACS (Picture Archiving Communication System) workstation (Centricity, GE Healthcare, Waukesha, WI) with a mediastinal window setting. Thymic enlargement was considered when both transverse and AP diameters increased. RTH was defined as the presence of thymic enlargement on CT performed after BMT relative to the previous $\mathrm{CT}$ taken before BMT. Thymus was also evaluated regarding morphological features such as triangular or nodular, well defined or irregular, homogeneous or heterogeneous, and other findings like calcification and necrosis. We investigated the associated risk factors for RTH. The patients with neoplastic disease involving the anterior mediastinum, who had undergone previous surgery and/or biopsy of mediastinum were excluded from the study. Approval was received from our Institutional Ethical Committee, and informed consent was obtained from the patients for the study. All procedures followed were in accordance with the ethical standards of the responsible committee on human experimentation and with the Helsinki Declaration of 1964 and later versions.

\section{Statistical methods}

Statistical analyses were performed using SPSS statistical software, version 19.0 for Windows (IBM Company, Chicago, IL, USA). Chi-square or Fisher exact tests were used to compare the categorical variables. A $p$ value $<0.05$ was considered statistically significant.

\section{Results}

Fifty-six patients who had different haemoto-oncological diseases with a total of 112 chest CT exams were included in the study. Twenty of 56 patients (36\%) demonstrated RTH (12 boys, 8 girls; age range $=4-18$ years; median age $=9.8$ years). In 20 patients with RTH, seven patients (35\%) were diagnosed with acute lymphoblastic leukaemia (ALL), five patients (25\%) with thalassaemia, two patients (10\%) with acute myeloid leukaemia (AML), and one patient (5\%) with non-Hodgkin's lymphoma (NHL), haemophagocytic syndrome, neuroblastoma, Fanconi anaemia, aplastic anaemia, and immune deficiency. Table 1 shows the pa-

Table 1. Patient characteristics

\begin{tabular}{|c|c|c|c|}
\hline Characteristic & RTH (+) & RTH (-) & $p$ \\
\hline age (years) & $9.8(4-18)$ & $11.2(3-18)$ & $>0.05$ \\
\hline $\operatorname{sex}(M: F)$ & $12: 8$ & $22: 14$ & $>0.05$ \\
\hline diagnosis & 20 & 36 & $>0.05$ \\
\hline ALL & 7 & 12 & \\
\hline thalassaemia & 5 & 9 & \\
\hline AML & 2 & 4 & \\
\hline $\begin{array}{l}\mathrm{NHL} \\
\text { haemophagocytic S } \\
\text { aplastic anaemia } \\
\text { immune deficiency }\end{array}$ & 1 & 2 & \\
\hline Fankoni anaemia & 1 & 1 & \\
\hline neuroblastoma & 1 & 0 & \\
\hline $\begin{array}{l}\text { rhabdomyosarcoma } \\
\text { Ewing sarcoma }\end{array}$ & 0 & 1 & \\
\hline
\end{tabular}

tient characteristics. All 20 patients (100\%) with RTH in the study were asymptomatic. At follow-up, mediastinal relapse tumour was not detected in any patient (0\%). The mean follow-up period between the time of CT taken before BMT and the time of BMT was 46 days (range 5-196 days). The time from BMT to CT performed after BMT was 239 days (range 101 to 481 days). While the mean AP diameter of the thymus was $9 \mathrm{~mm}$ and the mean transverse diameter was $16 \mathrm{~mm}$ before BMT, they were measured as $17 \mathrm{~mm}$ and $33 \mathrm{~mm}$, respectively, after BMT. Both diameters showed an almost $100 \%$ increase. The mean $\mathrm{HU}$ (Hounsfield Unit) value of the thymus was measured as 57 (range 33-81) on contrast enhanced images and 35 (range 8-64) on unenhanced images before BMT, which were 59 (range 39-78) on images with contrast and 36 (range 10-61) on images without contrast, respectively, after BMT. In all patients (100\%) with RTH, chest CT revealed a well-defined triangular shaped homogeneous soft tissue lesion without mass effect in the anterior mediastinum. Thymic nodularity, irregularity, heterogeneity, calcification, and necrosis were not detected in any patients (0\%). When comparing the characteristics between patients with and without RTH, no statistical correlation was found between RTH and age, sex, and diagnosis.

\section{Discussion}

RTH is known as the development of thymic enlargement after recovery from various stress factors. It indicates regeneration of the cell-mediated immune system and may represent a better prognosis in children with lymphoma [7]. A few studies have reported the incidence of RTH; most of them presented case reports or case series [8, 9]. Kissin et al. [10] found RTH in $11.6 \%$ of their patients with testicular teratomas after chemotherapy. Jeon et al. [11] reported the incidence of RTH as $1.49 \%$ after radioactive iodine ablation therapy in patients with thyroid cancer. Chen at al. [7] detected RTH in 21/31 (68\%) of their patients with lymphoma. Their interval between the ending of chemotherapy and diagnosis of RTH ranged from 1 month to 17 months, which was similar with the previous reports [9, 10] and this study (101 to 481 days). In this study, RTH was detected in 20/56 (36\%) of all patients and in 1/3 (33\%) of patients with $\mathrm{NHL}$, which was significantly lower than the ratios found by Chen et al. [7]. The percentages of RTH were $7 / 19(37 \%)$ in patients with ALL, $2 / 6$ (33\%) in AML, and $9 / 25(36 \%)$ in all patients with acute leukaemia. The ratio of $5 / 14$ (33\%) observed in patients with thalassaemia was similar to the rates of other diseases in this study.

Some studies suggest differentiation of RTH from lymphoma relapse with chest CT features. Yarom et al. [9] reported that triangular shaped, bilobulated, homogeneous soft tissue with convex contour in the normal thymic region refers to $\mathrm{RTH}$. In this study, all patients with $\mathrm{RTH}$ demonstrated well-defined, triangular shaped, homogeneous soft tissue without mass affect in the anterior mediastinum on chest CT (Figs. 1, 2). Araki et al. [5] found that lymphoid hyperplasia of the thymus revealed significantly higher CT attenuation than the true hyperplasia (RTH). They also observed pyramidal shape in $80 \%$ of the patients 

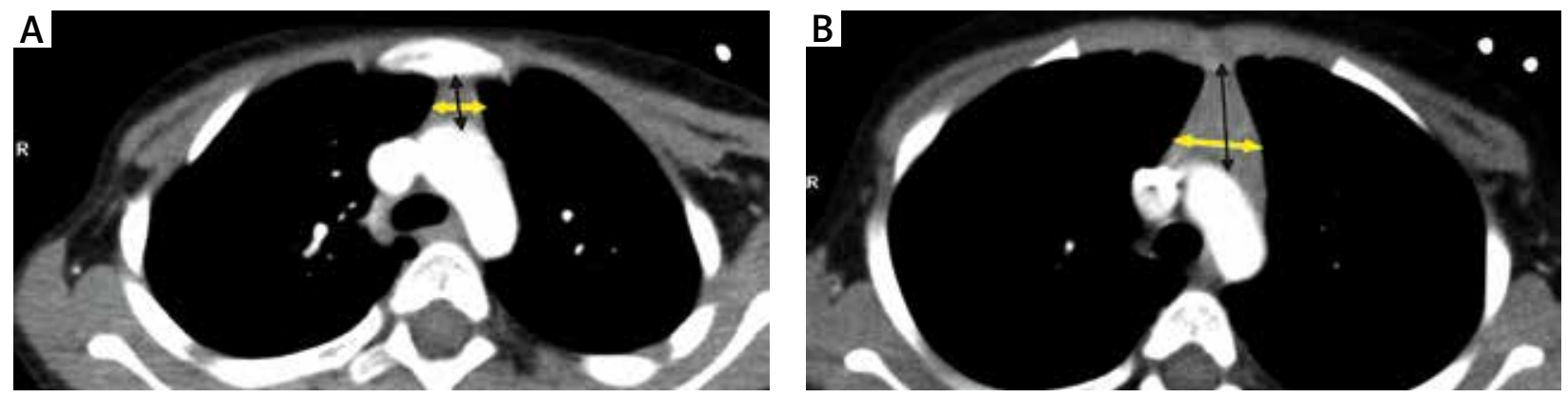

Fig. 1. Measurement of transverse (yellow arrows) and anterior-posterior (black arrows) diameters of thymus on contrast-enhanced axial CT images of the chest performed 22 days before (A) and 159 days after (B) bone marrow transplantation in a five-year-old girl with acute lymphoblastic leukaemia. Prominent increase is observed in both diameters after transplantation
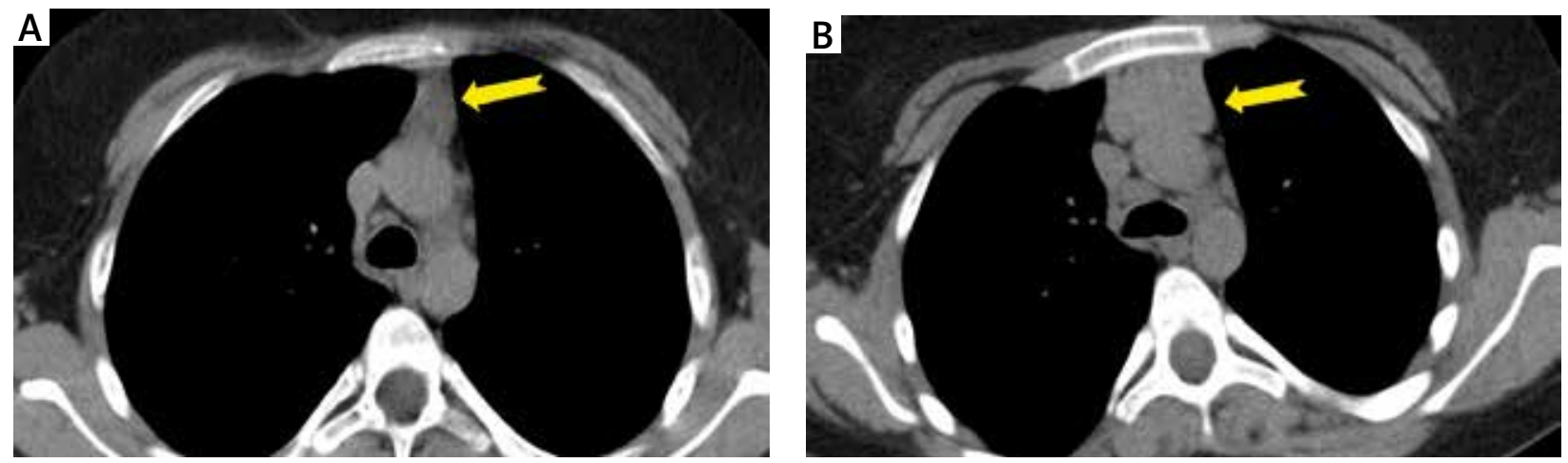

Fig. 2. 16-year-old girl with aplastic anaemia. Axial CT images without contrast performed 24 days before (A) and 282 days after (B) bone marrow transplantation show significant increase in the both diameters (yellow arrows) of the thymus after transplantation

as being the most common morphologic feature of thymic hyperplasia. In our study, the mean HU value of the thymus was 59 on contrast enhanced images and 36 on unenhanced images after BMT, which was significantly higher than the HU values, found as 31.4 and -1.6, respectively, by Araki et al. in adults with true hyperplasia, supporting the knowledge that CT attenuation of the thymus reduces with age due to the increasing of fat content [5]. They reported a mean $\mathrm{HU}$ value of 47.9 in patients with lymphoid hyperplasia, with a cutoff value of $41.2 \mathrm{HU}$ to differentiate lymphoid hyperplasia from true hyperplasia. There was no statistically significant difference between the $\mathrm{HU}$ values measured before and after BMT in our study.

PET-CT is a recent modality for lymphoma staging and follow-up, but it is not able to differ RTH from thymic tumours [11]. Thymus shows physiological FDG uptake in prepubertal age and sometimes in young adults [12]. Brink et al. [13] revealed that FDG uptake overlaps between normal thymus and thymic pathologies.

Magnetic resonance imaging (MRI) is a problem-solving tool for evaluation of the thymus. It is superior to CT in differentiating cystic from solid lesions and hyperplastic thymus from thymic neoplasms. Chemical-shift MRI including signal-intensity index and chemical-shift ratio have high accuracy in distinguishing thymic hyperplasia from tumours [14]. In their study of 41 patients with thymic lesions, Inaoka et al. [15] reported decreased signal on the out-of-phase MR images relative to the in-phase images in all 23 patients with thymic hyperplasia but in none of the 18 patients with thymic tumour. Priola et al. [16] and Ara- ki et al. [5] found similar findings that suggest dual-echo chemical-shift MRI was valuable in differentiation of thymic hyperplasia from thymic tumours. Diffusion-weighted MRI may be useful in differential diagnosis of non-suppressing cases on chemical-shift MRI by demonstrating restriction of diffusion in malignant lesions [17]. Bakan et al. [18] reported that CT perfusion is helpful in differentiating thymoma from lymphoma, thymic carcinoma, and invasive lung cancer involving the anterior mediastinum. Blood flow and blood volume values were higher in thymoma in comparison to thymic hyperplasia; however, the difference was not statistically significant.

Jeon et al. [11] found that the incidence of RTH significantly reduced from second to the fifth decade of life in their study. They also noted female predominance in patients with RTH. Chen et al. [7] observed that the mean age appeared to be lower in patients with RTH than in patients without RTH, and female patients appeared to be more likely to demonstrate RTH than male patients, but these findings were not statistically significant. No statistical correlation was found between RTH and possible risk factors in this study, which had several limitations, including its retrospective format, small number of patients, and irregular intervals of $\mathrm{CT}$ examinations. More comprehensive prospective studies are required to clarify the exact incidence of RTH and to identify the associated risk factors.

\section{Conclusions}

In conclusion, RTH is a common finding after BMT in children with various haemato-oncological diseases and 
should be taken under consideration in paediatric patients after BMT. Correct diagnosis is essential to prevent invasive procedures like chemotherapy, radiotherapy, biopsy, and surgery.

The authors declare no conflict of interests.

\section{References}

1. Küpeli S. Rebound thymic hyperplasia. Cukurova Med J 2017; 42: 799-800.

2. Araki T, Sholl LM, Gerbaudo VH, Hatabu H. Thymic measurements in pathologically proven normal thymus and thymic hyperplasia: Intra and interobserver variability. Acad Radiol 2014; 21: 733-742.

3. Ackman JB, Kovacina B, Carter BW, Wu CC, Sharma A, Shepard $J$-AO, Halpern EF. Sex difference in normal thymic appearance in adults 20-30 years of age. Radiology 2013; 268: 245-253.

4. Nishino M, Ashiku SK, Kocher ON, Thurer RL, Boiselle PM, Hatabu $H$. The thymus: a comprehensive review. RadioGraphics 2006; 26 : 335-348.

5. Araki T, Sholl LM, Gerbaudo VH, Hatabu H, Nishino M. Imaging characteristics of pathologically proven thymic hyperplasia: identifying features that can differentiate true from lymphoid hyperplasia. AJR Am J Roentgenol 2014; 202: 471-478.

6. Sfikakis PP, Gourgoulis GM, Moulopoulos LA, Kouvatseas G, Theofilopoulos AN, Dimopoulos MA. Age-related thymic activity in adults following chemotherapy-induced lymphopenia. Eur J Clin Invest 2005; 35: 380-387.

7. Chen CH, Hsiao CC, Chen YC, Ko SF, Huang SH, Huang SC, Hsieh KS, Sheen JM. Rebound Thymic Hyperplasia after Chemotherapy in Children with Lymphoma. Pediatr Neonatol 2017; 58: 151-157.

8. Bangerter M, Behnisch W, Griesshammer M. Mediastinal masses diagnosed as thymus hyperplasia by fine needle aspiration cytology. Acta Cytol 2000; 44: 743-777.

9. Yarom N, Zissin R, Apter S, Hertz M, Rahimi-Levene N, Gayer G. Rebound thymic enlargement on CT in adults. Int J Clin Pract 2007; 61: 562-588.

10. Kissin CM, Husband JE, Nicholas D, Eversman W. Benign thymic enlargement in adults after chemotherapy: CT demonstration. Radiology 1987; 163: 67-70.

11. Wittram C, Fischman AJ, Mark E, Ko J, Shepard JA. Thymic enlargement and FDG uptake in three patients: CT and FDG positron emission tomography correlated with pathology. AJR Am J Roentgenol 2003; 180: 519-522.

12. Nakahara T, Fujii H, Ide M, Nishiumi N, Takahashi W, Yasuda S, Shothsu A, Kubo A. FDG uptake in the morphologically normal thymus: comparison of FDG positron emission tomography and $\mathrm{CT}$. $\mathrm{Br}$ J Radiol 2001; 74: 821-824.

13. Brink I, Reinhardt MJ, Hoegerle S, Altehoefer C, Moser E, Nitzsche EU. Increased metabolic activity in the thymus gland studied with 18F-FDG PET: age dependency and frequency after chemotherapy. J Nucl Med 2001; 42: 591-595.

14. Ackman JB, Wu CC. MRI of the thymus. AJR Am J Roentgenol 2011; 197: 15-20.

15. Inaoka T, Takahashi K, Mineta M, et al. Thymic hyperplasia and thymus gland tumors: differentiation with chemical shift MR imaging. Radiology 2007; 243: 869-876.

16. Priola AM, Priola SM, Ciccone G, et al. Differentiation of rebound and lymphoid thymic hyperplasia from anterior mediastinal tumors with dual-echo chemical-shift MR imaging in adulthood: reliability of the chemical-shift ratio and signal intensity index. Radiology 2015; 274: 238-249.

17. Priola AM, Gned D, Marci V, Veltri A, Priola SM. Diffusion-weighted $M R I$ in a case of nonsuppressing rebound thymic hyperplasia on chemical-shift MRI. Jpn J Radiol 2015; 33: 158-163.

18. Bakan S, Kandemirli SG, Dikici AS, et al. Evaluation of anterior mediastinal solid tumors by CT perfusion: a preliminary study. Diagn Interv Radiol 2017; 23: 10-14.

\section{Address for correspondence}

\section{Taner Arpaci}

Department of Radiology

Acibadem Adana Hospital, Acibadem University

Cumhuriyet Caddesi No. 66,

01130, Seyhan, Adana, Turkey

e-mail: tanerarpaci@yahoo.com

Submitted: 17.03 .2018

Accepted: $\quad 8.04 .2018$ 